\title{
ON THE MACKEY PROBLEM FOR FREE LOCALLY CONVEX SPACES
}

\author{
SAAK GABRIYELYAN
}

\begin{abstract}
We show that the free locally convex space $L(X)$ over a Tychonoff space $X$ is a Mackey group iff $L(X)$ is a Mackey space iff $X$ is discrete.
\end{abstract}

\section{INTRODUCTION}

Let $(E, \tau)$ be a locally convex space (lcs for short). A locally convex vector topology $\nu$ on $E$ is called compatible with $\tau$ if the spaces $(E, \tau)$ and $(E, \nu)$ have the same topological dual space. The classical Mackey-Arens theorem states that for every lcs $(E, \tau)$ there exists the finest locally convex vector space topology $\mu$ on $E$ compatible with $\tau$. The topology $\mu$ is called the Mackey toplogy on $E$ associated with $\tau$, and if $\mu=\tau$, the space $E$ is called a Mackey space.

An analogous notion in the class of locally quasi-convex (lqc for short) abelian groups was introduced in [3]. For an abelian topological group $(G, \tau)$ we denote by $\widehat{G}$ the group of all continuous characters of $(G, \tau)$ (for all relevant definitions see the next section). Two topologies $\mu$ and $\nu$ on an abelian group $G$ are said to be compatible if $\widehat{(G, \mu)}=\widehat{(G, \nu)}$. Following [3], an lqc abelian group $(G, \mu)$ is called a Mackey group if for every lqc group topology $\nu$ on $G$ compatible with $\tau$ it follows that $\nu \leq \mu$.

Not every Mackey lcs is a Mackey group. In [6] we show that the space $C_{p}(X)$, which is a Mackey space for every Tychonoff space $X$, is a Mackey group if and only if it is barrelled. In particular, this result shows that there are even metrizable lcs which are not Mackey groups that gives a negative answer to a question posed in [4]. Only very recently, answering Question 4.4 of [5], Außenhofer [1] and the author [7] independently have shown that there are lqc groups which do not admit a Mackey group topology. For historical remarks, references and open questions we referee the reader to [5, 12. In Question 4.3 of [5], we ask: For which Tychonoff spaces $X$ the free lcs $L(X)$ is a Mackey space or a Mackey group? Below we give a complete answer to this question.

Theorem 1.1. For a Tychonoff space $X$, the following assertions are equivalent:

(i) $L(X)$ is a Mackey group;

(ii) $L(X)$ is a Mackey space;

(iii) $X$ is discrete.

In particular, Theorem 1.1 essentially strengthen Theorem 6.4 of [9] which states that $L(X)$ is quasibarrelled if and only of $X$ is discrete.

\section{Proof of Theorem 1.1}

We start from some necessary definitions and notations. Let $X$ be a Tychonoff space. The space $X$ is called a $k_{\mathbb{R}}$-space if every real-valued function on $X$ which is continuous on every compact subset of $X$ is continuous on $X$. A subset $A$ of $X$ is called functionally bounded in $X$ if every continuous real-valued function on $X$ is bounded on $A$, and $X$ is a $\mu$-space if every functionally bounded subset of $X$ has compact closure. The Dieudonné completion $\mu X$ of $X$ is always a $\mu$-space.

2000 Mathematics Subject Classification. Primary 46A03; Secondary 54H11.

Key words and phrases. free locally convex space, Mackey topology. 
Denote by $\mathbb{S}$ the unit circle group and set $\mathbb{S}_{+}:=\{z \in \mathbb{S}: \operatorname{Re}(z) \geq 0\}$. Let $G$ be an abelian topological group. A character $\chi \in \widehat{G}$ is a continuous homomorphism from $G$ into $\mathbb{S}$. A subset $A$ of $G$ is called quasi-convex if for every $g \in G \backslash A$ there exists $\chi \in \widehat{G}$ such that $\chi(g) \notin \mathbb{S}_{+}$ and $\chi(A) \subseteq \mathbb{S}_{+}$. The group $G$ is called locally quasi-convex if it admits a neighborhood base at the neutral element 0 consisting of quasi-convex sets. Every real locally convex space is a locally quasi-convex group by Proposition 2.4 of [2].

Following [11, the free locally convex space $L(X)$ on a Tychonoff space $X$ is a pair consisting of a locally convex space $L(X)$ and a continuous map $i: X \rightarrow L(X)$ such that every continuous map $f$ from $X$ to a locally convex space $E$ gives rise to a unique continuous linear operator $\bar{f}: L(X) \rightarrow E$ with $f=\bar{f} \circ i$. The free locally convex space $L(X)$ always exists and is essentially unique. The set $X$ forms a Hamel basis for $L(X)$ and the map $i$ is a topological embedding, see [13, 14,

Let $X$ be a Tychonoff space. For $\chi=a_{1} x_{1}+\cdots+a_{n} x_{n} \in L(X)$ with distinct $x_{1}, \ldots, x_{n} \in X$ and nonzero $a_{1}, \ldots, a_{n} \in \mathbb{R}$, we set

$$
\|\chi\|:=\left|a_{1}\right|+\cdots+\left|a_{n}\right|, \text { and } \operatorname{supp}(\chi):=\left\{x_{1}, \ldots, x_{n}\right\} .
$$

For an lcs $E$, we denote by $E^{\prime}$ the topological dual space of $E$. For a cardinal number $\kappa$, the classical Banach space $c_{0}(\kappa)$ consists of all bounded functions $g: \kappa \rightarrow \mathbb{R}$ such that the set $\{i \in \kappa:|g(i)| \geq \varepsilon\}$ is finite for every $\varepsilon>0$ and is endowed with the supremum norm $\|\cdot\|_{\infty}$.

We denote by $C_{k}(X)$ the space $C(X)$ of all real-valued continuous functions on $X$ endowed with the compact-open topology $\tau_{k}$. The support of a function $f \in C(X)$ is denoted by $\operatorname{supp}(f)$. Denote by $M_{c}(X)$ the space of all real regular Borel measures on $X$ with compact support. It is well-known that the dual space of $C_{k}(X)$ is $M_{c}(X)$, see [10, Proposition 7.6.4]. For every $x \in X$, we denote by $\delta_{x} \in M_{c}(X)$ the evaluation map (Dirac measure), i.e. $\delta_{x}(f):=f(x)$ for every $f \in C(X)$. Denote by $\tau_{e}$ the polar topology on $M_{c}(X)$ defined by the family of all equicontinuous pointwise bounded subsets of $C(X)$. We shall use the following deep result of Uspenskiı [14].

Theorem 2.1 (14]). Let $X$ be a Tychonoff space and let $\mu X$ be the Dieudonné completion of $X$. Then the completion $\overline{L(X)}$ of $L(X)$ is topologically isomorphic to $\left(M_{c}(\mu X), \tau_{e}\right)$.

We need also the following corollary of Theorem 2.1 noticed in [8].

Corollary $2.2([8])$. Let $X$ be a $\mu$-space. Then the topology $\tau_{e}$ on $M_{c}(X)$ is compatible with the duality $\left(C_{k}(X), M_{c}(X)\right)$.

Proof. It is well-known that $L(X)^{\prime}=C(X)$, see [13. Now Theorem 2.1 implies $\left(M_{c}(X), \tau_{e}\right)^{\prime}=$ $L(X)^{\prime}=C(X)$.

The next lemma follows from Proposition 2.5 of [5], we give its proof for the sake of completeness of the paper.

Lemma 2.3. If a real lcs $(E, \tau)$ is a Mackey group, then it is a Mackey space.

Proof. Let $\nu$ be a locally convex vector topology on $E$ compatible with $\tau$. Applying Proposition 2.3 of 2] we obtain $\widehat{(E, \nu)}=\widehat{(E, \tau)}$. Hence $\nu$ is a locally quasi-convex group topology (see Proposition 2.4 of [2]) compatible with $\tau$. Therefore $\nu \leq \tau$ since $(E, \tau)$ is a Mackey group. Thus $(E, \tau)$ is a Mackey space.

We need the following characterization of non-discrete Tychonoff spaces.

Proposition 2.4. A Tychonoff space $X$ is not discrete if and only if there exist an infinite cardinal $\kappa$, a point $z \in X$, a family $\left\{g_{i}\right\}_{i \in \kappa}$ of continuous functions from $X$ to $[0,2]$ and a family $\left\{U_{i}\right\}_{i \in \kappa}$ of open subsets of $X$ such that

(i) $\operatorname{supp}\left(g_{i}\right) \subseteq U_{i}$ for every $i \in \kappa$;

(ii) $U_{i} \cap U_{j}=\emptyset$ for all distinct $i, j \in \kappa$; 
(iii) $z \notin U_{i}$ for every $i \in \kappa$ and $z \in \operatorname{cl}\left(\bigcup_{i \in \kappa}\left\{x \in X: g_{i}(x) \geq 1\right\}\right)$.

Proof. The sufficiency follows from (i)-(iii) which cannot hold simultaneously for discrete spaces. To prove the necessity we consider two cases.

Case 1. There is a continuous function $h: X \rightarrow[0,1]$ such that the set $L:=\{x \in X: h(x)>0\}$ is not closed. So there is a $z \in \operatorname{cl}(L)$ such that $h(z)=0$. We distinguish between two subcases.

Subcase 1.1. For every neighborhood $W$ of $z$, the closure $\overline{h(W)}$ of $h(W)$ contains an interval of the form $[0, \varepsilon)$ for some $\varepsilon>0$. For every $n \in \mathbb{N}$, set

$$
t_{n}(x):=\max \left\{h(x)-\frac{1}{3 n+1}, 0\right\} \cdot \max \left\{0, \frac{1}{3 n-1}-h(x)\right\}
$$

and

$$
A_{n}:=\sup \left\{t_{n}(x): x \in X\right\} .
$$

Let $m$ be the least natural number such that $A_{n}>0$ for every $n>m$. For every $n>m$, set

$$
U_{n}:=h^{-1}\left(\frac{1}{3 n+1}, \frac{1}{3 n-1}\right) \text { and } g_{n}(x):=\frac{2}{A_{n}} \cdot t_{n}(x),
$$

Then, for every $n>m$, we have $g_{n}(X) \subseteq[0,2], \operatorname{supp}\left(g_{n}\right) \subseteq U_{n}$ and $z \notin U_{n}$. Clearly, (i) and (ii) are fulfilled and $z \notin U_{n}$ for every $n>m$. So to check that the sequences $\left\{U_{n}: n>m\right\}$ and $\left\{g_{n}: n>m\right\}$ and the point $z$ satisfy (i)-(iii) we have to show that $z \in \operatorname{cl}\left(\bigcup_{n>m} g_{n}^{-1}([1,2])\right)$.

Fix arbitrarily a neighborhood $W$ of $z$ in $X$. Then, by assumption, $\overline{h(W)}$ contains $[0, \varepsilon)$ for some $\varepsilon \in(0,1)$. Therefore, if $n_{0}>(1+3 \varepsilon) /(3 \varepsilon)$ there is a $y \in W$ such that $t_{n_{0}}(y) \geq(1 / 2) A_{n_{0}}$, and hence $g_{n_{0}}(y) \geq 1$. Thus $g_{n_{0}}^{-1}([1,2]) \cap W$ is not empty and hence $z \in \operatorname{cl}\left(\bigcup_{n>m} g_{n}^{-1}([1,2])\right)$.

Subcase 1.2. There is a neighborhood $W$ of $z$ such that the closure $\overline{h(W)}$ of $h(W)$ does not contain an interval of the form $[0, \varepsilon)$. Then there exist sequences $\left\{a_{n}\right\}_{n \in \mathbb{N}}$ and $\left\{b_{n}\right\}_{n \in \mathbb{N}}$ in $(0,1)$ converging to zero such that

$$
b_{n+1}<a_{n}<b_{n},\left[b_{n+1}, a_{n}\right] \cap \overline{h(W)}=\emptyset \text { and }\left(a_{n}, b_{n}\right) \cap \overline{h(W)} \neq \emptyset, \quad \forall n \in \mathbb{N} .
$$

Set $a_{0}:=1$ and

$$
c_{n}:=\frac{1}{2}\left(b_{n+1}+a_{n}\right) \text { and } d_{n}:=\frac{1}{2}\left(b_{n}+a_{n-1}\right), \quad \forall n \in \mathbb{N} .
$$

Then $c_{n}<a_{n}<b_{n}<d_{n}<1$. For every $n \in \mathbb{N}$, let $r_{n}(x)$ be the piecewise linear continuous function from $[0,1]$ to $[0,1]$ such that

$$
r_{n}\left(\left[0, c_{n}\right] \cup\left[d_{n}, 1\right]\right)=\{0\} \text { and } r_{n}\left(\left[a_{n}, b_{n}\right]\right)=\{1\},
$$

and set

$$
U_{n}:=h^{-1}\left(c_{n}, d_{n}\right) \text { and } g_{n}(x):=r_{n}(h(x)), \quad x \in X .
$$

By construction, the sequences $\left\{U_{n}: n \in \mathbb{N}\right\}$ and $\left\{g_{n}: n \in \mathbb{N}\right\}$ and the point $z$ satisfy (i) and (ii) and $z \notin U_{n}$ for every $n \in \mathbb{N}$. Let us show that every neighborhood $U$ of $z$ contains elements of $\bigcup_{n \in \mathbb{N}} g_{n}^{-1}(\{1\})$. We can assume that $U \subseteq W$. Since $\left[b_{n+1}, a_{n}\right] \cap \overline{h(W)}=\emptyset$ we obtain that $h(W) \subseteq\{0\} \cup \bigcup_{n \in \mathbb{N}}\left(a_{n}, b_{n}\right)$. Therefore, if $y \in U$ and $n_{0} \in \mathbb{N}$ is such that $h(y) \in\left(a_{n_{0}}, b_{n_{0}}\right)$ (such a $y$ exists because $z \in \mathrm{cl}(L))$, then $g_{n_{0}}(y)=1$.

Case 2. For every continuous function $h: X \rightarrow[0,1]$ the set $\{x \in X: h(x)>0\}$ is closed.

We claim that $X$ has a neighborhood base containing closed-and-open sets. Indeed, since $X$ is Tychonoff, for every point $x \in X$ and each open neighborhood $U$ of $x$ there is a continuous function $h: X \rightarrow[0,1]$ such that $h(x)=1$ and $h(X \backslash U)=\{0\}$. It remains to note that, by assumption, the open neighborhood $h^{-1}((0,1]) \subseteq U$ of $x$ also is closed.

Now, by the assumption of the proposition, there is a non-isolated point $z \in X$. By the Zorn lemma, there exists a maximal (under inclusion) family $\mathcal{U}=\left\{U_{i}: i \in \kappa\right\}$ of pairwise disjoint closed-and-open sets such that $z \notin U_{i}$ for every $i \in \kappa$. The maximality of $\mathcal{U}$ and the claim imply 
S. GABRIYELYAN

that $z \in \operatorname{cl}(\cup \mathcal{U})$. For every $i \in \kappa$, let $g_{i}$ be the characteristic function of $U_{i}$. Clearly, the families $\mathcal{U}$ and $\left\{g_{i}: i \in \kappa\right\}$ and the point $z$ satisfy conditions (i)-(iii).

The following proposition is crucial for the proof of Theorem 1.1 .

Proposition 2.5. Let $X$ be a Dieudonné complete space. If $\left(M_{c}(X), \tau_{e}\right)$ is a Mackey space, then $X$ is discrete.

Proof. Suppose for a contradiction that $X$ is not discrete. Then, by Proposition 2.4, there exist an infinite cardinal $\kappa$, a point $z \in X$, a family $\left\{g_{i}\right\}_{i \in \kappa}$ of continuous functions from $X$ to $[0,2]$ and a family $\left\{U_{i}\right\}_{i \in \kappa}$ of open subsets of $X$ satisfying (i)-(iii) of that proposition. Define a map $R: M_{c}(X) \rightarrow c_{0}(\kappa)$ by

$$
R(\mu):=\left(\mu\left(g_{i}\right)\right), \quad \mu \in M_{c}(X) .
$$

Claim 1. The map $R$ is well-defined.

Indeed, let $\mu \in M_{c}(X)$ be a positive measure. Since $\mu$ is finite and $\sigma$-additive, the condition (ii) of Proposition 2.4 implies that for every $\varepsilon>0$ the number of indices $i \in \kappa$ for which $\mu\left(U_{i}\right) \geq \varepsilon$ is finite. Now the claim follows from the inclusion $\operatorname{supp}\left(g_{i}\right) \subseteq U_{i}$ (see (i)) and the inequalities $0 \leq \mu\left(g_{i}\right) \leq 2 \mu\left(U_{i}\right)$.

Consider a map $T: M_{c}(X) \rightarrow\left(M_{c}(X), \tau_{e}\right) \times c_{0}(\kappa)$ defined by

$$
T(\mu):=(\mu, R(\mu)), \quad \forall \mu \in M_{c}(X) .
$$

The map $T$ is well-defined by Claim 1 . Denote by $\mathcal{T}$ the locally convex vector topology on $M_{c}(X)$ induced from the product $\left(M_{c}(X), \tau_{e}\right) \times c_{0}(\kappa)$.

Claim 2. The topology $\mathcal{T}$ is compatible with $\tau_{e}$.

First we note that for every $\left(\lambda_{i}\right) \in\left(c_{0}(\kappa)\right)^{\prime}=\ell_{1}(\kappa)$, the function $\sum_{i} \lambda_{i} g_{i}$ belongs to $C(X)$. The Hahn-Banach extension theorem implies that every $\chi \in\left(M_{c}(X), \mathcal{T}\right)^{\prime}$ has the form

$$
\chi=\left(F,\left(\lambda_{i}\right)\right) \text {, where } F \in\left(M_{c}(X), \tau_{e}\right)^{\prime} \text { and }\left(\lambda_{i}\right) \in \ell_{1}(\kappa) .
$$

By Corollary 2.2, we have $F \in C(X)$ and hence $G:=F+\sum_{i \in \kappa} \lambda_{i} f_{i} \in C(X)$. Therefore

$$
\chi(\mu)=\mu(F)+\sum_{i \in \kappa} \lambda_{i} \cdot \mu\left(g_{i}\right)=\mu\left(F+\sum_{i \in \kappa} \lambda_{i} g_{i}\right)=\mu(G), \forall \mu \in M_{c}(X) .
$$

Applying Corollary 2.2 once again we obtain $\chi=G \in\left(M_{c}(X), \tau_{e}\right)^{\prime}$ as desired.

Claim 3. We claim that $\tau_{e}<\mathcal{T}$.

Indeed, it is clear that $\tau_{e} \leq \mathcal{T}$. Set

$$
S:=\left\{\delta_{x}: \text { there is an } i \in \kappa \text { such that } g_{i}(x) \geq 1\right\} \subseteq M_{c}(X) .
$$

To show that $\tau_{e} \neq \mathcal{T}$, we shall prove that (1) $\delta_{z} \in \operatorname{cl}_{\tau_{e}}(S)$, and $(2) \delta_{z} \notin \operatorname{cl}_{\mathcal{T}}(S)$.

To prove that $\delta_{z} \in \mathrm{cl}_{\tau_{e}}(S)$, fix arbitrarily a standard neighborhood

$$
[K ; \varepsilon]:=\left\{\mu \in M_{c}(X):|\mu(f)|<\varepsilon \forall f \in K\right\}
$$

of zero in $\left(M_{c}(X), \tau_{e}\right)$, where $K$ is a pointwise bounded equicontinuous subset of $C(X)$ and $\varepsilon>0$. Choose a neighborhood $U$ of $z$ such that

$$
|f(x)-f(z)|<\varepsilon, \quad \forall f \in K, \quad \forall x \in U .
$$

By (iii) of Proposition 2.4, take an $i_{0} \in \kappa$ and $x_{i_{0}} \in U$ such that $g_{i_{0}}\left(x_{i_{0}}\right) \geq 1$. Then $\delta_{x_{i_{0}}} \in S$ and

$$
\left|\left(\delta_{x_{i_{0}}}-\delta_{z}\right)(f)\right|=\left|f\left(x_{i_{0}}\right)-f(z)\right|<\varepsilon, \quad \forall f \in K .
$$

Thus $\delta_{x_{i_{0}}} \in \delta_{z}+[K ; \varepsilon]$ and hence $\delta_{z} \in \operatorname{cl}_{\tau_{e}}(S)$.

To show that $\delta_{z} \notin \operatorname{cl}_{\mathcal{T}}(S)$, consider the neighborhood $W:=M_{c}(X) \times U$ of zero in $\mathcal{T}$, where $U=\left\{g \in c_{0}(\kappa):\|g\|_{\infty} \leq 1 / 2\right\}$. Fix arbitrarily $\delta_{x} \in S$ and choose $j \in \kappa$ such that $g_{j}(x) \geq 1$. Then 
the $j$ th coordinate $\delta_{x}\left(g_{j}\right)$ of $R\left(\delta_{x}\right)$ satisfies the following (in the last equality we use (i) and (ii) of Proposition 2.4)

$$
\left|\delta_{x}\left(g_{j}\right)-\delta_{z}\left(g_{j}\right)\right|=\left|g_{j}(x)-g_{j}(z)\right|=g_{j}(x) \geq 1>1 / 2 .
$$

Therefore $R\left(\delta_{x}\right)-R\left(\delta_{z}\right) \notin U$ and hence $\delta_{x}-\delta_{z} \notin W$. As $x$ was arbitrary we obtain $\delta_{z} \notin \operatorname{cl} \mathcal{T}(S)$.

Finally, Claims 2 and 3 imply that $\left(M_{c}(X), \tau_{e}\right)$ is not a Mackey space. This contradiction shows that $X$ must be discrete.

Theorem 1.1 follows from the next more general result.

Theorem 2.6. For a Tychonoff space $X$ the following assertions are equivalent:

(i) $L(X)$ is a Mackey group;

(ii) $L(X)$ is a Mackey space;

(iii) $\left(M_{c}(\mu X), \tau_{e}\right)$ is a Mackey group;

(iv) $\left(M_{c}(\mu X), \tau_{e}\right)$ is a Mackey space;

(v) $X$ is discrete.

Proof. (i) $\Rightarrow$ (ii) and (iii) $\Rightarrow$ (iv) follow from Lemma 2.3 .

(ii) $\Rightarrow$ (iv) It is well known that the completion of a Mackey space is a Mackey space, see Proposition 8.5.8 of [10]. Therefore, by Theorem 2.1, the space $\left(M_{c}(\mu X), \tau_{e}\right)$ is a Mackey space.

(iv) $\Rightarrow(\mathrm{v})$ By Proposition 2.5, $\mu X$ is discrete. Thus $X$ is discrete as well.

$(\mathrm{v}) \Rightarrow(\mathrm{i})$,(iii) Since $X$ is discrete Theorem 2.1 implies $L(X)=\left(M_{c}(X), \tau_{e}\right)$. Therefore $L(X)$ is a barrelled space by [9, Theorem 6.4], and hence $L(X)$ is a Mackey group by [3, Theorem 4.2].

\section{REFERENCES}

1. L. Außenhofer, On the non-existence of the Mackey topology for locally quasi-convex groups, Forum Math. accepted.

2. W. Banaszczyk, Additive subgroups of topological vector spaces, LNM 1466, Berlin-Heidelberg-New York 1991.

3. M.J. Chasco, E. Martín-Peinador, V. Tarieladze, On Mackey topology for groups, Studia Math. 132 (1999), $257-284$.

4. D. Dikranjan, E. Martín-Peinador, V. Tarieladze, Group valued null sequences and metrizable non-Mackey groups, Forum Math. 26 (2014), 723-757.

5. S. Gabriyelyan, On the Mackey topology for abelian topological groups and locally convex spaces, Topology Appl. 211 (2016), 11-23.

6. S. Gabriyelyan, A characterization of barrelledness of $C_{p}(X)$, J. Math. Anal. Appl. 439 (2016), $364-369$.

7. S. Gabriyelyan, A locally quasi-convex abelian group without a Mackey group topology, Proc. Amer. Math. Soc., accepted.

8. S. Gabriyelyan, Maximally almost periodic groups and respecting properties, available in arXiv:1712.05521

9. S.S. Gabriyelyan, S.A. Morris, Free topological vector spaces, Topology Appl., 223 (2017), 30-49.

10. H. Jarchow, Locally Convex Spaces, B.G. Teubner, Stuttgart, 1981.

11. A.A. Markov, On free topological groups, Dokl. Akad. Nauk SSSR 31 (1941), 299-301.

12. E. Martín-Peinador, V. Tarieladze, Mackey topology on locally convex spaces and on locally quasi-convex groups. Similarities and historical remarks, Revista de la Real Academia de Ciencias Exactas, Fisicas y Naturales. Serie A. Matematicas RACSAM, 110 (2016), 667-679.

13. D.A. Raı̌kov, Free locally convex spaces for uniform spaces, Math. Sb. 63 (1964), 582-590.

14. V.V. Uspenskiı̌, Free topological groups of metrizable spaces, Math. USSR-Izv. 37 (1991), 657-680.

Department of Mathematics, Ben-Gurion University of the Negev, Beer-Sheva, P.O. 653, Israel E-mail address: saak@math.bgu.ac.il 